\title{
Management of Established Status Epilepticus
}

\author{
Phil E. Smith, M.D.
}

Status epilepticus is a medical emergency characterized either by continued seizures or by a lack of full recovery between seizures. It is relatively common, with a reported annual incidence of between 10 and 41 cases per 100,000 population (the variation partly reflects differing diagnostic criteria) and a mortality of approximately $20 \%{ }^{1}$ The previous definition of status epilepticus as epileptic activity persisting for more than $30 \mathrm{~min}$ utes has been superseded ${ }^{2}$ with the aim of limiting the irreversible neurologic damage that has been shown in animal models. ${ }^{3}$ Clinicians have long been urged instead to intervene early in the disorder, typically when seizures have persisted beyond 5 minutes. ${ }^{4}$ Adherence to standard evidence-based management protocols that include this approach has been shown to improve outcomes of status epilepticus in adults and children, ${ }^{5}$ whereas delaying such intervention can allow seizures to persist and to become refractory, with risk of neurologic harm and death, particularly from generalized tonic-clonic status epilepticus.

Management protocols divide the medical therapy for status epilepticus into three main stages. The first-line treatment for early status epilepticus, often administered before the patient reaches the hospital, is a benzodiazepine ${ }^{6}$ - usually intravenous lorazepam or buccal midazolam. However, up to a third of cases are resistant to benzodiazepines. The second-line medication if status epilepticus becomes established is any one of the available intravenous preparations: fosphenytoin (a precursor drug to phenytoin), levetiracetam, or sodium valproate given by infusion, usually in the hospital. Observational and retrospective data have suggested that valproate ${ }^{7}$ or levetiracetam ${ }^{8}$ has an advantage in efficacy, but there is insufficient evidence for the preference of one over another. In the absence of highquality data, the choice of drug depends on this ambiguous evidence regarding the efficacy and side-effect profiles, on the availability and cost of the drugs, and on regulatory approval.

Third-line treatment for refractory status epilepticus, which is usually managed in an inten- sive care unit, is not supported by high-quality evidence to guide the choice of one of three anesthetic agents: midazolam, propofol, or thiopental. It is unclear whether earlier intervention with anesthetics in status epilepticus could improve outcomes at this stage, and we need further studies to address this issue. A fourth stage of "super-refractory" status epilepticus - ongoing seizures despite general anesthesia - has practically no meaningful evidence to support various desperate pharmacologic, immunologic, dietary, and surgical measures that have been tried on an individual basis.

The Established Status Epilepticus Treatment Trial (ESETT), described in this issue of the Jour$n a l,{ }^{9}$ has assessed the relative efficacies and sideeffect profiles of the intravenous medications used as second-line therapy for status epilepticus. The trial essentially showed that intravenous levetiracetam, fosphenytoin, and valproate each led to approximately $50 \%$ of patients having seizure cessation at 1 hour, with similar percentages of patients in the three treatment groups having adverse events. There was a trend toward a shorter time to seizure termination favoring valproate - 7.0 minutes, as compared with 10.5 minutes for levetiracetam and 11.7 minutes for fosphenytoin - but these times could be analyzed only in the small subgroup of 39 patients (10\%) for whom audio recordings had enabled accurate timing. It has been a commendable achievement to deliver a multicenter, randomized, double-blind trial of a potentially lifesaving intravenous medication to 384 patients within highpressure emergency department settings, including explaining concepts of clinical equipoise, randomization, and consent to distressed caregivers and relatives of an unconscious, convulsing patient. Even the diagnosis of status epilepticus itself is challenging, since this is still made mostly on clinical grounds and is sometimes incorrect: $10 \%$ of the patients enrolled into ESETT were subsequently found to have dissociative (psychogenic, nonepileptic) seizures. However, the pragmatic decision not to require electroencephalographic (EEG) monitoring for inclusion 
in the trial reflects the limited availability of this technology and of its expert interpretation. We need further studies to determine the benefits of more widespread application of EEG technology for the diagnosis and monitoring of status epilepticus.

The ESETT results may consolidate the drug management protocols for established (benzodiazepine-resistant) status epilepticus. Having three equally effective second-line intravenous medications means that the clinician may choose a drug that takes into account individual situations that may be modified by factors such as the presumed underlying cause of status epilepticus; coexisting conditions, including allergy, liver and renal disease, hypotension, propensity to cardiac arrhythmia, and alcohol and drug dependence; the currently prescribed antiepileptic treatment; the cost of the medication; and governmental agency drug approval.

The ESETT trialists have provided a service to adults and children with status epilepticus and to the teams managing their conditions. Providing evidence underpinning such an important treatment decision has the potential to save lives and brain tissue. But the practical challenge for the management of status epilepticus remains the same as in the past: ensuring that clinicians are familiar with, and follow, a treatment protocol.

Disclosure forms provided by the author are available with the full text of this editorial at NEJM.org.

From the University Hospital of Wales, Cardiff, United Kingdom.

1. Betjemann JP, Lowenstein DH. Status epilepticus in adults. Lancet Neurol 2015;14:615-24.

2. Trinka E, Cock H, Hesdorffer D, et al. A definition and classification of status epilepticus - report of the ILAE Task Force on Classification of Status Epilepticus. Epilepsia 2015;56:151523.

3. Meldrum BS, Brierley JB. Prolonged epileptic seizures in primates: ischemic cell change and its relation to ictal physiological events. Arch Neurol 1973;28:10-7.

4. Lowenstein DH, Alldredge BK. Status epilepticus. N Engl J Med 1998;338:970-6.

5. Jones S, Pahl C, Trinka E, Nashef L. A protocol for the inhospital emergency drug management of convulsive status epilepticus in adults. Pract Neurol 2014;14:194-7.

6. Silbergleit R, Durkalski V, Lowenstein D, et al. Intramuscular versus intravenous therapy for prehospital status epilepticus. N Engl J Med 2012;366:591-600.

7. Alvarez V, Januel J-M, Burnand B, Rossetti AO. Second-line status epilepticus treatment: comparison of phenytoin, valproate, and levetiracetam. Epilepsia 2011;52:1292-6.

8. Misra UK, Kalita J, Maurya PK. Levetiracetam versus lorazepam in status epilepticus: a randomized, open labeled pilot study. J Neurol 2012;259:645-8.

9. Kapur J, Elm J, Chamberlain JM, et al. Randomized trial of three anticonvulsant medications for status epilepticus. N Engl J Med 2019;381:2103-13.

DOI: 10.1056/NEJMe1913775

Copyright (c) 2019 Massachusetts Medical Society. 